\title{
Divergências entre histórico da consulta e diagnóstico médico de agressóes físicas registradas nos prontuários de uma unidade de urgência e emergência
}

I 1 Cléa Adas Saliba Garbin, ${ }^{2}$ Ana Paula Dossi de Guimarães e Queiroz, ${ }^{3}$ Tânia Adas Saliba Rovida, ${ }^{4}$ Artênio José Isper Garbin I

Resumo: Inúmeros podem ser os indicadores da violência na sociedade. Contudo, em nenhum outro serviço de saúde a violência adquire tamanha visibilidade quanto na emergência. Este estudo teve por objetivo analisar se há divergência entre o histórico da consulta e o diagnóstico médico de agressões físicas atendidas no pronto-socorro. Realizou-se estudo transversal no serviço de urgência e emergência de Araçatuba-SP, com base em prontuários médicos. Consideraram-se os dados dos pacientes, lesões, histórico, diagnóstico e tratamento. Dos 133.537 atendimentos, somente 153 foram registrados como agressão física, apesar de outros 161 trazerem a informação sobre violência no histórico da consulta; 59,6\% eram homens e 60,6\% tinham entre 20 e 44 anos. Predominaram escoriaçōes, dor e ferimentos corto-contusos. Não foram encontradas associações entre "mencionar violência no diagnóstico" e as características dos pacientes e atendimentos (horário, encaminhamento, gênero, faixa etária). Conclui-se que, na maioria dos casos, a violência relatada no histórico da consulta não foi mencionada no diagnóstico das lesões. As características do atendimento e dos pacientes não estiveram associadas ao fato de os profissionais diagnosticarem o caso como violência.

> Palavras-chave: violência; atitude do pessoal de saúde; saúde pública; serviços médicos de emergência.
${ }^{1}$ Departamento de Odontologia Infantil e Social Universidade Estadual Júlio de Mesquita Filho (Unesp), São Paulo, Brasil. Endereço eletrônico: cgarbin@ foa.unesp.br

${ }^{2}$ Departamento de Odontologia Infantil e Social da Unesp, São Paulo, Brasil. Endereço eletrônico: anadossi@foa. unesp.br

${ }^{3}$ Departamento de Odontologia Infantil e Social da Unesp, São Paulo, Brasil. Endereço eletrônico: tasalibarovida@foa. unesp.br

${ }^{4}$ Departamento de Odontologia Infantil e Social da Unesp, São Paulo, Brasil. Endereço eletrônico: agarbin@foa. unesp.br
Recebido em: 05/07/2012 Aprovado em: 19/08/2013 
A violência é atualmente um dos mais graves problemas de saúde pública no Brasil e em diversos outros países (GOMES et al., 2002). Estima-se que, a cada ano, as causas violentas sejam responsáveis por mais de um milhão de mortes no mundo (DAHLBERG; KRUG, 2008; DESLANDES, 1999; DOSSI et al., 2008; DOSSI, 2009; GARBIN et al., 2006; GAWRYSZEWSKI et al., 2006; GOMES et al., 2002; HOFNER et al., 2005; IBGE, 2011; KRUG et al., 2003; LEAL; LOPES, 2005; MASCARENHAS et al., 2009). Inúmeros podem ser os indicadores desse fenômeno na sociedade. Contudo, em nenhum outro serviço de saúde a violência adquire tamanha visibilidade quanto na emergência. Esta representa, sem dúvida, a principal porta de entrada desses casos no sistema público de saúde (DESLANDES, 1999).

A morbimortalidade gerada por situaçōes violentas significa uma demanda importante a ser considerada na formulação de políticas voltadas ao setor. Sendo assim, é de se esperar que os casos referentes a agressões sejam diagnosticados e componham estatística específica, promovendo o conhecimento do assunto por gestores e demais responsáveis. Nessa perspectiva, a informação deve ser encarada como um direito da sociedade, e não como um produto de uso privado de instituições, sejam elas científicas, burocráticas ou de comunicação. Quando bem gerada, sistematizada e divulgada, a informação pode mediar os processos de conscientização e promover a integração dos setores segmentados da sociedade (NJAINE et al., 1997).

Os sistemas de informações disponíveis no Sistema Único de Saúde (SUS) são estratégicos na definição de prioridades (QUEIROZ et al., 2009). No Brasil, o monitoramento da violência para fins epidemiológicos é realizado por meio da análise dos dados da declaração de óbito (DO) e da autorização de internação hospitalar (AIH), fornecidos pelo Sistema de Informações sobre Mortalidade (SIM) e pelo Sistema de Informações Hospitalares do Sistema Único de Saúde (SIH/SUS) (MASCARENHAS et al., 2009; NJAINE; REIS, 2005). Esse monitoramento, entretanto, apresenta limitaçôes, já que esses dados são restritos aos casos que resultem em óbito ou internação da vítima.

$\mathrm{Na}$ tentativa de retratar adequadamente a real conjuntura, a captação e a preservação desses dados têm sido incentivadas. Em 2006, o Ministério da Saúde 
(VIVA), com a finalidade de viabilizar a obtenção de dados e informaçôes sobre violências não fatais e suas vítimas (MASCARENHAS et al., 2009).

Todavia, aparentemente, ainda não há uma mobilização geral. O sistema de captação de informações não abrange todas as unidades de saúde; falta envolvimento dos serviços e profissionais responsáveis. Assim, o atendimento ao usuário em um serviço de emergência prossegue voltado à lesão causada e não inclui um olhar à violência, não identificada como causa do trauma (LEAL; LOPES, 2005). Dessa forma, é provável que essas situaçôes sejam subestimadas e os dados disponíveis não reflitam a realidade. É possível que, entre as razões para essa subestimação, estejam fatores relacionados tanto às características da ocorrência (horário, dia da semana, fluxo intenso de atendimento) quanto aos profissionais (experiência, formação, conhecimento, disponibilidade, percepção, entre outras).

A abordagem da violência pela saúde pública deve basear-se no maior número possível de conhecimentos básicos de todos os seus aspectos e unir, sistematicamente, dados de sua extensão e características locais, nacionais e internacionais (KRUG et al., 2003; MASCARENHAS et al., 2009).

Considerando a necessidade de se caracterizar a demanda gerada por violências no âmbito hospitalar, aliada ao fato de que as informações de mortalidade e morbidade nesse setor são limitadas, foi proposto este estudo, que teve por objetivo analisar se há divergência entre o histórico da consulta e o diagnóstico médico de agressões físicas atendidas em uma unidade de urgência e emergência em 2008.

\section{Metodologia}

Um estudo transversal foi realizado no município de Araçatuba, estado de São Paulo, que possui uma população estimada de 178.598 habitantes (IBGE, 2011) e conta com três unidades públicas de atendimento a urgências e emergências. Foi desenvolvido no Pronto-Socorro Municipal Aida Vanzo Dolce. O local foi escolhido por ser sede do SAMU (Serviço de Atendimento Móvel de Urgência) e concentrar o departamento de estatística em saúde da cidade. Para o início da pesquisa, foi obtida autorização do secretário de saúde do município e da responsável técnica pela referida unidade. 
A princípio, pretendia-se utilizar os dados contidos no sistema digital da instituição. Contudo, uma análise prévia demonstrou que nem todos os casos de agressão eram lançados como tal, uma vez que os funcionários baseiamse no diagnóstico e no código da Classificação Internacional de Doenças (CID-10) escritos pelos médicos. Caso haja história de agressão física, mas o diagnóstico mencione somente a lesão ocorrida, este será o dado considerado. Dessa forma, a coleta de dados baseou-se nos prontuários médicos dos pacientes atendidos no período de janeiro a dezembro de 2008, e foi realizada por uma única pesquisadora. Todos os 133.537 registros foram avaliados. Compuseram a amostra aqueles que, no histórico da consulta, se referiam a situações de agressão física independentemente do diagnóstico.

Foram considerados os dados relativos à identificação dos pacientes, características das lesões, histórico, diagnóstico e tratamento. Ressalta-se que as informações dos documentos analisados eram limitadas, principalmente no que se refere às características do paciente. Além disso, especial dificuldade foi encontrada quanto à caligrafia dos médicos. Nesses casos, os funcionários do setor de estatística, responsáveis pela digitação das fichas, auxiliaram a interpretação.

Os dados foram digitados e analisados com auxílio do software Epi Info 3.5.1. Para verificação de associação entre as variáveis, utilizou-se o teste estatístico Qui-quadrado e, na impossibilidade de sua aplicação, o Exato de Fisher.

Atendendo aos preceitos éticos da Resolução 196/96, o projeto foi encaminhado para avaliação pelo Comitê de Ética em Pesquisa da Faculdade de Odontologia de Araçatuba (FOA-Unesp) e aprovado sob o número 2007-01343.

\section{Resultados}

Foram realizados 133.537 atendimentos entre $1^{\circ}$ de janeiro e 31 de dezembro de 2008. Destes, 153 casos (48,7\%) foram registrados no banco de dados da instituição como "agressão por meio de força corporal", conforme os achados deste estudo. Outros 161 prontuários (51,3\%) informavam a violência como causa das lesões no campo destinado ao histórico da consulta (figura 1). No total, representaram 314 consultas ou $0,23 \%$ de todos os atendimentos. Das 314 consultas por violências, duas foram autoinfligidas, e as outras 312, provocadas por terceiros. Não foi registrado nenhum caso de óbito por agressão nos documentos analisados. 
física, segundo a inclusão no banco de dados institucional como "agressão por meio de força corporal". Araçatuba, SP, 2008

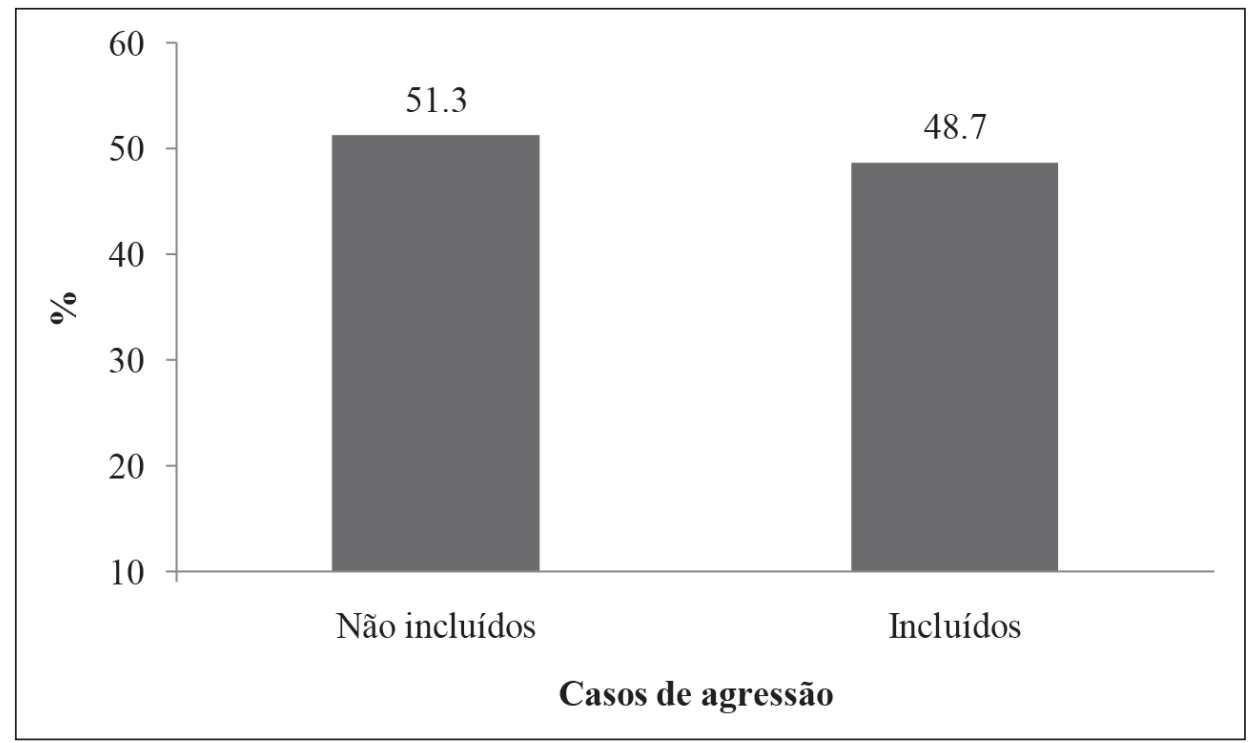

A respeito da natureza da violência, $12,7 \%$ dos casos foram praticados por familiares, 2,9\% oriundos de assaltos, 1,0\% praticados durante o trabalho, 1,0\% na escola e $0,3 \%$ pela polícia. Os demais $258(82,1 \%)$ prontuários traziam apenas exposições vagas sobre o histórico da agressão, o que impossibilita a descrição precisa dessa variável. Quanto ao gênero, 59,6\% dos pacientes atendidos eram homens e $40,4 \%$, mulheres.

A figura 2 retrata as frequências conforme a faixa etária. O número de adultos entre 20 e 44 anos perfaz mais que uma vez e meia o total de pacientes das outras faixas etárias. 

faixa etária. Araçatuba, SP, 2000

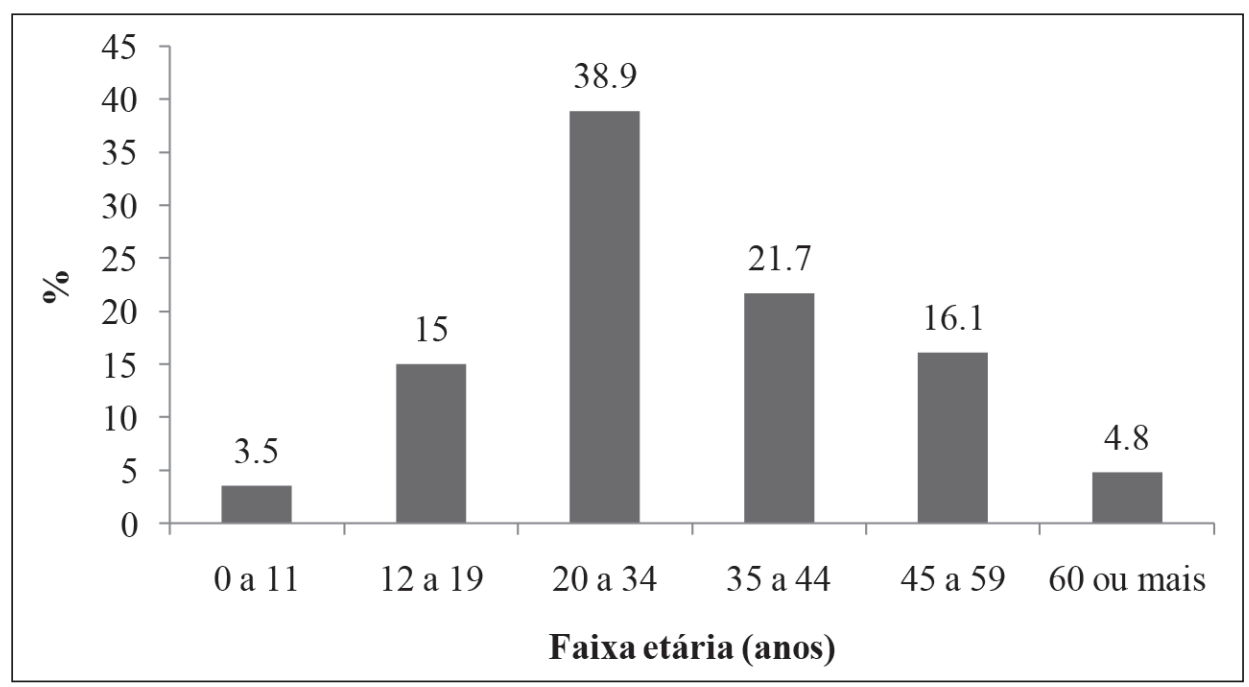

Os atendimentos desse tipo de ocorrência ocorreram com maior frequência no período das 18 às 6 horas (55,8\%), 15,1\% a mais que o total dos outros horários, conforme ilustra a figura 3. $62,4 \%$ dos atendimentos foram realizados entre segundas e sextas-feiras, enquanto $37,6 \%$ ocorreram aos sábados ou domingos.

Figura 3. Distribuição percentual dos atendimentos das vítimas de agressão física, segundo o horário. Araçatuba, SP, 2008

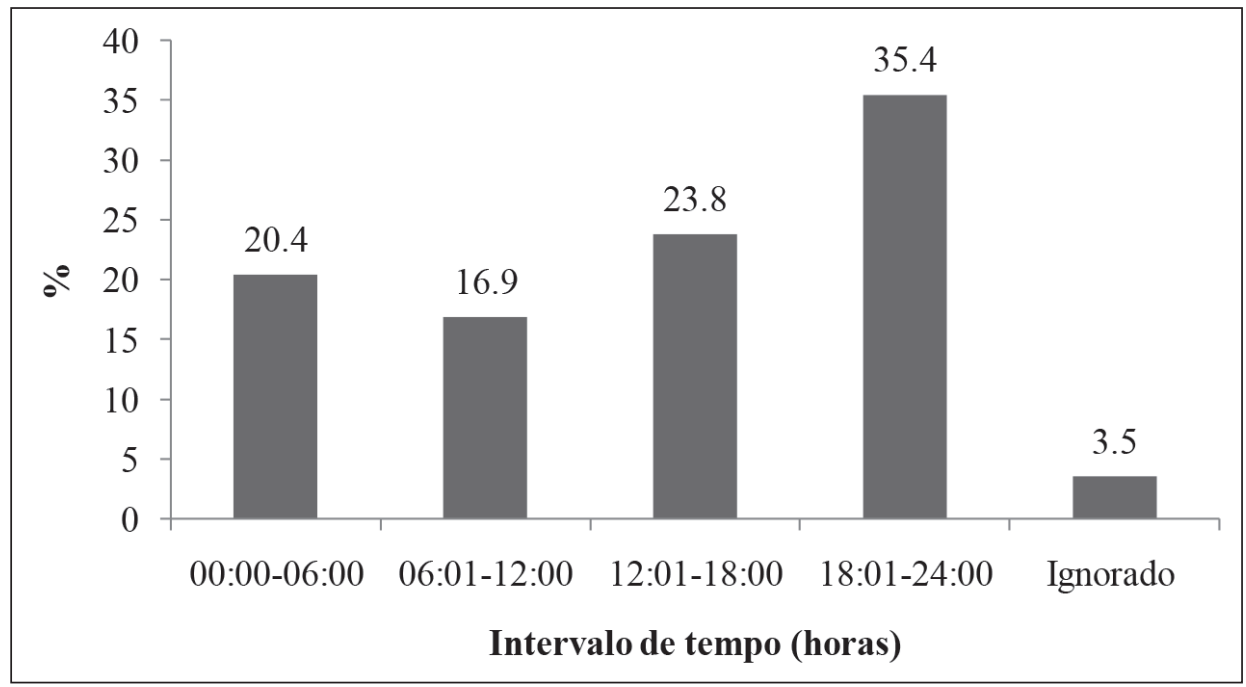


Analisando-se a tabela 1, pode-se verificar que os sinais e sintomas apresentados

foram, com maior frequência: escoriaçōes, dor, ferimentos corto-contusos e hematomas. As altas taxas de ferimentos não especificados pelos médicos (15,4\%) impedem uma descrição mais precisa dessas características.

Tabela 1. Distribuição percentual dos sinais, sintomas e locais diagnosticados no momento da consulta médica. Araçatuba, SP, 2008

\begin{tabular}{|c|c|c|}
\hline Características & $\mathbf{n}$ & $\%$ \\
\hline \multicolumn{3}{|l|}{ Sinal/Sintoma* } \\
\hline Escoriação & 85 & 17,6 \\
\hline Dor & 59 & 12,3 \\
\hline Ferimento corto-contuso & 50 & 10,3 \\
\hline Hematoma & 44 & 9,2 \\
\hline Edema & 37 & 7,6 \\
\hline Ferimento contuso & 30 & 6,2 \\
\hline Ferimento inciso & 25 & 5,2 \\
\hline Equimose & 19 & 4,0 \\
\hline Fratura & 12 & 2,5 \\
\hline Sangramento & 11 & 2,3 \\
\hline Outros & 36 & 7,4 \\
\hline Ferimento não especificado & 75 & 15,4 \\
\hline Total & 483 & 100,0 \\
\hline \multicolumn{3}{|l|}{ Locais* } \\
\hline Cabeça/pescoço & 226 & 50,8 \\
\hline Membros superiores & 111 & 24,9 \\
\hline Tórax/dorso & 56 & 12,6 \\
\hline Membros inferiores & 31 & 7,0 \\
\hline Quadril/pelve & 14 & 3,1 \\
\hline Abdome & 7 & 1,6 \\
\hline Total & 445 & 100,0 \\
\hline
\end{tabular}

*Poderiam ser apontados mais de um sinal/sintoma e local lesionados por paciente. 
Foram constatados 633 procedimentos realizados pelos profissionais, além da consulta médica, como exame radiográfico, sutura, administração de medicamentos, entre outros. Em média, foram descritos dois procedimentos suplementares à consulta.

Os dados referentes às características dos pacientes e atendimentos foram cruzados com a variável "mencionar violência no diagnóstico". Não foram encontradas associações relativas ao horário de atendimento $\left(\chi^{2}=2,491\right.$, $\mathrm{p}=0,1145, \mathrm{gl}=1)$, gênero do paciente $\left(\chi^{2}=5,966, \mathrm{p}=0,0145, \mathrm{gl}=1\right)$, sua faixa etária $\left(\chi^{2}=1,5603, \mathrm{p}=0,9060, \mathrm{gl}=5\right)$ e necessidade de encaminhamento (teste Exato de Fisher, $\mathrm{p}=1,0000)$.

\section{Discussão}

$\mathrm{O}$ atendimento de emergência é um poderoso indicador da violência que ocorre na cidade (DESLANDES, 1999). Entretanto, para que retrate fielmente a situação, é necessário que seus registros sejam confiáveis e bem elaborados. A melhoria da qualidade da informação é um dos elementos fundamentais para dar mais precisão aos processos de tomada de decisão, sobretudo em relação a um tema tão crucial e urgente para a sociedade brasileira como a violência (NJAINE; REIS, 2005).

Uma das críticas que se faz à ficha de atendimento no serviço pesquisado diz respeito aos dados sociodemográficos do paciente. Coletados na recepção, eles são escassos e mal identificam o sujeito. Sabe-se que a formulação de estratégias de combate aos problemas de saúde pública depende em parte da caracterização dos grupos prioritários, a qual, conforme evidenciado neste estudo, ficou prejudicada.

Assim, as únicas informaçôes relativas às pessoas atendidas que puderam ser extraídas desses documentos foram gênero e idade. Quanto ao gênero, prevaleceram os homens. A faixa etária predominante foi a de adultos jovens, o que pode estar relacionado a fatores como a busca por emoções e riscos típica dessa fase da vida. Outra hipótese pode estar assentada no fato de que, provavelmente, a violência urbana, responsável pela maior vitimização de homens (SOUZA; LIMA, 2006), tenha ocorrido na maioria dos casos. Todavia, uma análise mais precisa a esse respeito ficou prejudicada, uma vez que poucos prontuários indicavam a natureza da agressão. 
Esses achados estão de acordo com outros estudos. Porém, destaca-se que as mulheres podem ter buscado o serviço, mas omitido as reais causas das lesões. Bruschi et al. (2006), ao investigarem os locais mais procurados por vítimas de violência doméstica, verificaram que os serviços de saúde só foram buscados em 5,3\% dos casos. Da mesma forma, Schraiber et al. (2005), relatam que 15,6\% das vítimas pesquisadas recorreram ao serviço de saúde. Isso pode significar que as lesões não representaram um problema grave para as vítimas, ou que estas não consideraram o setor de saúde como competente para lidar com a violência sofrida (DOSSI et al., 2008).

Verificou-se que os atendimentos desse tipo de ocorrência foram predominantemente noturnos, em consonância com outra investigação efetuada no município a partir de dados policiais (DOSSI et al., 2008). Deslandes (1999), no entanto, destacou, em pesquisa realizada em serviços de emergência, que os plantóes diurnos foram os mais procurados pelas vítimas de violência, exceção feita aos finais de semana.

A respeito das lesões e locais atingidos, mais uma vez confirma-se a informação de que a região de cabeça e pescoço é a mais prevalente nesse tipo de ocorrência (DESLANDES, 1999; DOSSI et al., 2008; GARBIN et al., 2006). Os principais sinais e sintomas descritos nos prontuários foram escoriações, dores e ferimentos corto-contusos.

As consequências da violência, dentre outros aspectos, evidenciam-se no aumento de gastos com emergência, assistência e reabilitação, muito mais custosos que a maioria dos procedimentos médicos convencionais (MINAYO, 2005). Dentre os procedimentos realizados, destacaram-se os exames e a utilização de medicamentos, o que pode ter representado gastos consideráveis ao sistema de saúde. Para Sarti (2005), no contexto da emergência, dada a forma como se dá a identificação dos profissionais com esse atendimento, não há como não medicalizar a violência.

Embora o índice de pacientes atendidos por agressão física seja considerado pequeno se comparado aos encontrados em outros serviços (HOFNER et al., 2005), é importante observar que as informaçôes foram aferidas de acordo com a descrição médica dada ao caso. Provavelmente, muitas lesóes provenientes de situações violentas foram "mascaradas" e atribuídas a acidentes; ou ainda, mesmo quando relatadas pelos pacientes, podem não ter sido especificadas pelo 
profissional como provenientes de agressão, limitando-se aos termos "trauma", "ferimento" ou "lesão". Assim, o planejamento de estratégias efetivas fica comprometido frente à pouca representatividade dos dados.

Em pouco mais da metade dos casos analisados por este estudo, foi a negligência da informação no diagnóstico a responsável pela subestimação das ocorrências. Como citado anteriormente, a qualificação do sistema de informações como um todo é fundamental para se gerar dados que avaliem constantemente a situação dos agravos violentos (GAWRYSZEWSKI et al., 2006). É necessário que os profissionais se atentem à urgência de preservar e compartilhar essas informações, uma vez que constituem o "olhar" dos programas e gestores do sistema de saúde no serviço. Para muitas pessoas, o atendimento emergencial é a única oportunidade de estar, enquanto vítima, diante de um profissional de saúde (um representante do poder público), e, em muitos casos, esse é um dos poucos momentos em que a violência será declarada (DESLANDES, 1999).

A omissão não somente repercute nos bancos de dados do sistema, que deixam de ser sustentados adequadamente, mas anula e inviabiliza qualquer ação voltada à transformação, seja da realidade daquela única vítima, seja da situação de toda a sociedade. Nesse contexto, seria a vítima duplamente agredida, dessa vez pela violência institucional imposta pelos órgãos burocráticos do serviço público.

Assim, há que se levar em conta que os danos, as lesões, os traumas e as mortes causados por acidentes e violências correspondem a altos custos emocionais e sociais e gastos com aparatos de segurança pública. Além disso, acarretam prejuízos econômicos por causa dos dias de ausência no trabalho, pelos danos mentais e emocionais incalculáveis que provocam nas vítimas e em suas famílias e pelos anos de produtividade ou de vida perdidos (MINAYO, 2005).

Segundo informações obtidas junto à coordenação da unidade, os profissionais não recebem qualquer orientação a respeito do preenchimento do prontuário, contando somente com a liberdade que lhes é peculiar; ou seja, é o médico quem decide pelo preenchimento da forma que achar mais conveniente. Realmente, pôde-se verificar uma grande diversidade entre os profissionais, sendo alguns prontuários preenchidos detalhadamente e outros, de forma bastante sucinta.

Prevalece a omissão da violência nos registros. Mesmo o que é registrado padece de sérias limitações, tendo em vista o mau preenchimento dos formulários, que deixam de informar dados essenciais ao esclarecimento dos eventos, prejudicando a 
resolução dos casos (NJAINE; REIS, 2005). As hipóteses para essa conduta seriam, entre outras, a formação precária dos profissionais que lidam com os registros e a desvalorização desses dados nas instituições públicas. Somam-se a essas explicações o provável desconhecimento dos profissionais acerca da conduta a ser adotada frente aos casos e a falta de comprometimento com a questão (DOSSI, 2009; SALIBA et al., 2007). Nessa conjuntura, fica a dúvida se uma capacitação no intuito de sensibilizar os profissionais sobre o valor da informação não seria necessária.

Ainda que não se tenha a pretensão de acreditar que os serviços públicos de saúde tenham toda a responsabilidade no combate à violência, a eles cabe o envolvimento institucional e a capacitação de seus trabalhadores para esse enfrentamento (LEAL; LOPES, 2005). Portanto, é fundamental integrar os profissionais de maneira a torná-los a espinha dorsal das redes intersetoriais para a assistência e proteção das vítimas (REICHENHEIM et al., 2011).

Importante ressaltar que, neste estudo, não foram encontradas associações entre as características das ocorrências e dos pacientes e o ato de diagnosticar o caso como agressão. Nessa circunstância, a atitude dos profissionais pode estar relacionada somente a suas características pessoais de gênero, idade, formação e conhecimento sobre o assunto, o que merece ser analisado em investigaçóes futuras, uma vez que essa pesquisa limitou-se à análise dos prontuários.

A esse respeito, Sarti (2005) verificou, junto às equipes médicas de atendimento emergencial, que, geralmente, os profissionais tendem a reconhecer como violência apenas os casos envolvendo crianças, mulheres e idosos no âmbito familiar. Às vítimas de ferimentos por armas de fogo ou armas brancas - em maioria homens e jovens - não é atribuída a vulnerabilidade; por isso, não são vistos como vítimas da violência, mesmo constituindo a maior parte delas.

Alguns autores afirmam que contribuem para a má qualidade dos prontuários as péssimas condições de trabalho e o despreparo dos profissionais com relação à importância da atividade que realizam (NJAINE; REIS, 2005).

\section{Conclusões}

$\mathrm{Na}$ maioria dos casos, a violência relatada no histórico da consulta não foi mencionada no diagnóstico das lesōes. As vítimas foram em maioria homens, prevalecendo os adultos jovens, e os atendimentos foram predominantemente noturnos. Pôde-se verificar que os sinais e sintomas apresentados com maior 
frequência foram: escoriaçōes, dor, ferimentos corto-contusos e hematomas. As características do atendimento e dos pacientes não estiveram associadas ao fato de os profissionais diagnosticarem o caso como violência. ${ }^{1}$

Dada a situação apresentada, é imprescindível uma atuação profissional crítica diante da violência, que questione os relatos de "fatalidade" e que não a trate exclusivamente como uma demanda de atendimento médico ao trauma (DESLANDES, 1999). Também fica a ideia de que são necessárias ações voltadas à sensibilização e à capacitação profissional para a correta elaboração do prontuário de pacientes, em especial aqueles vitimados pela violência, de forma a contribuir para o conhecimento da amplitude dessas situações na sociedade.

\section{Referências}

BRUSCHI, A.; PAULA, C.S. de; BORDIN, I.A.S. Prevalência e procura de ajuda na violência conjugal física ao longo da vida. Rev Saúde Pública, São Paulo, v.40, n.2, p.256-264, 2006.

DAHLBERG, L.L.; KRUG, E.G. Violence, a global public health problem. Ciênc. Saúde Coletiva, Rio de Janeiro, v.11, supl., p.277-292, 2006.

DESLANDES, S.F. O atendimento às vítimas de violência na emergência: "prevenção numa hora dessas?”. Ciênc. Saúde. Coletiva, Rio de Janeiro, v.4, n.1, p.81-94, 1999.

DOSSI, A.P. et al. Perfil epidemiológico da violência física intrafamiliar: agressões denunciadas em um município do Estado de São Paulo, Brasil, entre 2001 e 2005. Cad. Saúde Pública, Rio de Janeiro, v.24, n.8, p.1939-1952, 2008.

DOSSI, A.P. Violência contra a criança: formação, conhecimento, percepção e atitude de profissionais da saúde e da educação. 2009. 87 p. (Doutorado em Saúde Coletiva) Faculdade de Odontologia de Araçatuba, Universidade Estadual Paulista, Araçatuba, 2009. GARBIN, C. A. S. et al. Violência doméstica: análise das lesões em mulheres. Cad. Saúde Pública, Rio de Janeiro, v.22, n.12, p.2567-2573, 2006.

GAWRYSZEWSKI, V.P. et al. A mortalidade por causas externas no Estado de São Paulo em 2005. Bepa [periódico on-line], v.33, n.3, p.2-7, 2006. Disponível em URL:http://www. cve.saude.sp.gov/agencia/bepa.htm. Acesso em: set 2011.

GOMES, R. et al. Por que as crianças são maltratadas? Explicações para a prática de maustratos infantis na literatura. Cad. Saúde Pública, Rio de Janeiro, v.18, n.3, p.707-714, 2002. HOFNER, M.C. et al. Prevalence of victims of violence admitted to an emergency department. Emerg Med J., v.22, p.481-485, 2005.

INSTITUTO BRASILEIRO DE GEOGRAFIA E ESTATÍSTICA. Disponível em: URL: http://www.ibge.gov.br/cidadesat/default.php. Acesso em: 30 set 2011. 
KRUG, E.G. et al. Informe mundial sobre la violencia y la salud. Washington, DC: OPAS, 2003. 62 p.

LEAL, S.M.C.; LOPES, M.J.M. A violência como objeto da assistência em um hospital de trauma: "o olhar" da enfermagem. Ciênc. Saúde Coletiva, Rio de Janeiro, v.10, n.2, p.419431, 2005.

MASCARENHAS, M.D.M. et al. Perfil epidemiológico dos atendimentos de emergência por violência no Sistema de Serviços Sentinela de Vigilância de Violências e Acidentes (Viva) - Brasil, 2006. Epidemiol. Serv. Saúde, Brasília, v.18, n.1, p.17-28, 2009.

MINAYO, M.C.S. Violência: um problema para a saúde dos brasileiros. In: BRASIL. Ministério da Saúde. Secretaria de Vigilância em Saúde. Impacto da violência na saúde dos brasileiros. Brasília: MS, 2005. p.9-41.

NJAINE, K.; REIS, A.C. Qualidade da informação sobre acidentes e violências. In: BRASIL. Ministério da Saúde. Secretaria de Vigilância em Saúde. Impacto da violência na saúde dos brasileiros. Brasília: MS, 2005. p.313-340.

NJAINE, K. et al. A produção de desinformação sobre violência: análise de uma prática discriminatória. Cad. Saúde Pública, Rio de Janeiro, v.13, n.3, p.405-414, 1997.

QUEIROZ, O.V. et al. A construção da Base Nacional de Dados em Terapia Renal Substitutiva (TRS) centrada no indivíduo: relacionamento dos registros de óbitos pelo subsistema de Autorização de Procedimentos de Alta Complexidade (Apac/SAI/SUS) e pelo Sistema de Informaçôes sobre Mortalidade (SIM) - Brasil, 2000-2004. Epidemiol. Serv. Saúde, Brasília, v.18, n.2, p.107-120, 2009.

REICHENHEIM, M.E. et al. Violência e lesões no Brasil: efeitos, avanços alcançados e desafios futuros. The Lancet [periódico on-line], p.75-89, 2011. Disponível em: URL:http// www.thelancet.com. DOI:10.1016/S0140 6736(11)60053-6. Acesso em: 9 maio 2011.

SALIBA, O. et al. Responsabilidade do profissional de saúde sobre a notificação de casos de violência doméstica. Rev. Saúde Pública, São Paulo, v.41, n.3, p.472-477, 2007.

SARTI, C.A. O atendimento de emergência a corpos feridos por atos violentos. Physis: Rev. Saúde Coletiva, Rio de Janeiro, v.15, n.1, p.107-126, 2005.

SCHRAIBER, L.B. et al. Violência dói e não é direito: a violência contra a mulher, a saúde e os direitos humanos. São Paulo: Unesp, 2005. 183 p.

SOUZA, E.R.; LIMA, M.L.C. The panorama of urban violence in Brazil and its capitals. Ciênc. Saúde Coletiva, Rio de Janeiro, v.11, n.2, p.363-373, 2006.

\section{Nota}

${ }^{1}$ C.A.S. Garbin participou da idealização do estudo, análise dos dados, redação e correção do artigo; A.P.D.G. Queiroz participou da idealização do estudo, coleta e análise dos dados, redação e correção do artigo; T.A.S. Rovida participou da idealização do estudo, análise dos dados, redação do artigo; e A.J.I. Garbin participou da idealização do estudo, análise dos dados, redação do artigo. 


\section{Abstract}

\section{Differences between history on consultation and medical diagnosis of physical aggressions registered in medical records of urgency and emergency units}

There can be several indicators of violence in society. However, in no other health unit such violence acquires visibility as in emergency. This study aimed to examine whether there is divergence between the history of medical consultation and diagnosis of physical aggressions in the emergency unit. A cross-sectional study was conducted in an emergency unit in the city of Araçatuba, state of São Paulo, Brazil, based on medical records, considering data on patients, lesions, history, diagnosis and treatment. Out of 133,537 visits, only 153 were recorded as physical aggressions, and 161 informed violence in the history of the consultation; 59.6\% were male, $60.6 \%$ were between 20 and 44 years old. Excoriations, pain and injury predominated. There were no associations between state violence in the diagnosis and the characteristics of patients and visits (schedule, routing, gender, age). The conclusion is that in most cases violence reported in the history of the consultation was not mentioned in the diagnosis of injuries. The characteristics of care and patients were not related to the fact that professionals diagnosed the case as violence.

> Key words: violence; attitude of health personnel; public health; emergency medical services. 